\title{
ATIVIDADE FÍSICA HABITUAL E RISCO CARDIOVASCULAR NA PÓS-MENOPAUSA
}

\author{
Raimunda Beserra da Silva*, lúcia Costa-Paiva, Aarão Mendes Pinto Neto, Angélica de Assunção Braga, Sirlei Siani Morais \\ Trabalho realizado no Departamento de Tocoginecologia da Faculdade de Ciências Médicas (FCM)/ Universidade Estadual de Campinas \\ (Unicamp), Campinas, SP
}

\author{
*Correspondência: \\ Rua Piratininga, 23, apto. \\ 24 Chácara da Barra \\ Campinas, São Paulo \\ Cep: 13092-670 \\ ray@fcm.unicamp.br
}

\begin{abstract}
RESUMO
OBjetivo. Avaliar a prevalência de atividade física habitual e risco cardiovascular em mulheres na pós-menopausa.

Métodos. Estudo de coorte transversal com 162 mulheres, entre 40 e 65 anos, em amenorréia há no mínimo 12 meses, acompanhadas no Ambulatório de Menopausa do CAISM/UNICAMP. As mulheres responderam oralmente o questionário International Physical Activity Questionnaire para avaliação do nível de atividade física, que abordou a freqüência e duração das atividades ocorridas durante uma semana normal, realizadas no transporte, trabalho, em casa e no lazer, classificando-as em sedentárias, insuficientemente ativas, ativas e muito ativas. Foram realizados exames laboratoriais para dosagem sérica de colesterol total, lipoproteína de alta densidade, lipoproteína de baixa densidade, triglicérides, glicemia de jejum e medidas de pressão arterial sistólica e diastólica.

Resultados. A média de idade das mulheres foi de 56,5 anos e idade na menopausa de 46 anos. A prevalência de atividade física foi de $83,3 \%$, sendo que 2,5\% foram classificadas como muito ativas, $80,8 \%$ ativas e $16,7 \%$ insuficientemente ativas. A maioria realizava atividades, principalmente no transporte e em casa, e apenas $38,3 \%$ realizavam atividade física durante 0 lazer. Nesta população, 87,7\% das mulheres apresentavam escore de Framingham inferior a 10, considerado de baixo risco cardiovascular.
\end{abstract}

Conclusä́o. Mulheres na pós-menopausa apresentam alta prevalência de atividade física habitual e baixa aderência ao exercício físico. O risco cardiovascular foi baixo na população estudada. É importante incentivar e orientar esta população a praticar atividade física compatível com suas condições físicas, estimulando a prática não somente da atividade física habitual, mas também do exercício físico.

Unitermos: Atividade motora. Exercício. Risco cardiovascular. Pós-menopausa.

\section{INTRODUÇÃO}

Atualmente, com o uso de tecnologias, tem havido uma importante redução da atividade física no trabalho, em casa e durante o lazer, ocasionando um estilo de vida sedentário para a maioria das populações. Estudos epidemiológicos têm mostrado que os índices de sedentarismo são altos, independentemente do sexo e da faixa etária ${ }^{1,2,3}$.

Nos EUA, 54,6\% da populaçãa adulta não realizam o mínimo de atividade necessário para a promoção da saúde 2 . No Brasil, na cidade do Rio de Janeiro, aproximadamente $60 \%$ dos homens e $78 \%$ das mulheres não praticam atividade física durante o lazer ${ }^{3}$, enquanto no Estado de São Paulo 46,5\% da população é considerada insuficientemente ativa ${ }^{4}$. Nas regiões Sudeste e Nordeste, apenas 13\% das pessoas relataram realizar atividade física por pelo menos 30 minutos em um ou mais dias da semana!.

Pesquisas mostram que o exercício físico pode ajudar na prevenção da obesidade ${ }^{5}$, diabetes ${ }^{5}$, dislipidemias ${ }^{5}$, doenças cardíacas $^{6}$ e hipertensão arterial ${ }^{7}$, enquanto a atividade física habitual, que engloba todos os movimentos realizados no cotidiano, também pode ter papel importante na prevenção de doenças cardiovasculares e redução dos sintomas da doença já instalada ${ }^{8}$.

O Center for Disease Control and Prevention (CDC) e o American College of Sports Medicine(ACSM) recomendam que, para a promoção da saúde, indivíduos devem realizar atividade física de intensidade moderada, pelo menos 30 minutos por dia, na maior parte dos dias da semana, de preferência todos, de forma contínua ou acumulada?. Essas também são as recomendações da American Heart Association (AHA) como uma das estratégias para a prevenção das doenças cardiovasculares ${ }^{8}$.

A Associação Médica Brasileira (AMB), o Conselho Federal de Medicina (CFM) e a Sociedade Brasileira de Medicina do Esporte (SBME), em posicionamento oficial sobre o tema "Atividade Física e Saúde", entre outras recomendações, estabelecem que os profissionais da saúde devem combater a inatividade física, estimulando o incremento da atividade física por meio de atividades informais e formais ${ }^{10}$. Mais especificamente sobre a saúde da mulher, a SBME recomenda o aumento da atividade física cotidiana, a inserção de exercícios físicos ou de práticas desportivas para ajudar no combate ao risco da doença arterial coronariana".

Sabe-se que as alterações hormonais que ocorrem no período da menopausa podem associar-se a algumas doenças ${ }^{12}$ que, aliadas a alterações na capacidade respiratória ocasionadas pelo aumento da idade cronológica ${ }^{13}$, podem contribuir ainda mais para a diminuição dos níveis de atividade física. Estudo sobre a prevalência da inatividade física na população do Estado do Rio Grande do Sul verificou que a 
proporção de mulheres sedentárias entre 20 e 29 anos foi de 38\%, aumentando de acordo com a faixa etária, chegando a $57 \%$ em mulheres acima dos 70 anos $^{14}$. Em mulheres na pós-menopausa, pouco se sabe sobre a prática de atividade física e sobre os padrões de comportamento desta prática realizados no transporte, no trabalho, em casa e no lazer. Isto dificulta a atuação dos profissionais da saúde em relação à orientação da prática de atividade adequada e direcionada a mulheres nesta faixa etária, visando a melhora da aptidão física e promoção da saúde.

O objetivo deste estudo foi avaliar a prevalência de atividade física habitual, principais tipos de atividades realizadas e risco cardiovascular em mulheres na pós-menopausa.

\section{Métodos}

Foi realizado um estudo de coorte transversal com 162 mulheres acompanhadas no Ambulatório de Menopausa do Centro de Atenção Integral à Saúde da Mulher (CAISM) da Universidade Estadual de Campinas (Unicamp). Os critérios para elegibilidade no estudo foram idade entre 40 e 65 anos, estar em amenorréia há no mínimo 12 meses, e aceitar participar da pesquisa, assinando o Termo de Consentimento Livre e Esclarecido. Este estudo foi aprovado pelo Comitê de Ética em Pesquisa da Faculdade de Ciências Médicas/Unicamp.

O cálculo amostral foi baseado no estudo de Matsudo et al. ${ }^{4}$, que observaram que aproximadamente $46 \%$ das mulheres entre 50 e 69 anos de idade no Estado de São Paulo eram sedentárias e insuficientemente ativas. Considerando essa freqüência como uma proporção multinomial, concluiu-se que I 25 mulheres eram necessárias para estimativas de frequêencias da atividade física nesta faixa etária, com precisões de 10\% de margem de erro e intervalos de confiança de $95 \%{ }^{15}$.

A avaliação do nível de atividade física foi realizada utilizando-se o questionário International Physical Activity Questionnaire(IPAQ), elaborado por pesquisadores de diversos países com suporte da Organização Mundial da Saúde (OMS), como parte de um estudo multicêntrico envolvendo 12 países, para conhecer a classificação da população em relação à atividade físicac ${ }^{16}$. Cada país participante adaptou e validou seu questionário, levando em consideração as características da população. No Brasil, este questionário foi validado pelo Centro de Estudos do Laboratório de Aptidão Física de São Caetano do Sul (CELAFISCS) ${ }^{17}$.

A coleta dos dados foi feita por meio de entrevista pessoal durante consulta ambulatorial, em que se aplicava o questionário que avaliou a freqüência, em dias, e a duração, em minutos, das atividades realizadas por mais de dez minutos contínuos durante uma semana normal. Neste trabalho, utilizou-se a versão longa do questionário, que inclui quatro componentes de atividade física: atividade física no trabalho, atividade física como meio de transporte, atividade física em casa (trabalho, tarefas domésticas e cuidados com a família) e atividade física no lazer (exercício físico, esporte, recreação), classificadas em vigorosas, moderadas e caminhada, de acordo com a intensidade, segundo o relato das mulheres. A classificação do nível de atividade foi obtida somando-se a freqüência e duração de todas as atividades, categorizando posteriormente as mulheres segundo o nível de atividade física em sedentárias, insuficientemente ativas, ativas e muito ativas ${ }^{17}$.

Para os exames laboratoriais, foram coletados $10 \mathrm{ml}$ de sangue

\section{Tabela I - Distribuição percentual das mulheres segundo o nível} de atividade física habitual $(\mathrm{n}=162)$

\begin{tabular}{|c|c|c|}
\hline Nível de atividade & $\mathbf{n}$ & $\%$ \\
\hline Muito ativa & $\overline{04}$ & $\overline{2,5}$ \\
\hline Ativa & $|3|$ & 80,8 \\
\hline Insuficientemente ativa & 27 & 16,7 \\
\hline Sedentária & - & - \\
\hline Total & 162 & 100 \\
\hline
\end{tabular}

venoso, em sistema fechado a vácuo, pela manhãa, após 10 - 12 horas de jejum. A análise foi realizada por meio do método enzimático colorimétrico para dosagem do perfil lipídico e glicose oxidase para dosagem da glicemia de jejum, utilizando o analisador automático Hitachi 917. As pressões arteriais (PA) sistólica e diastólica foram medidas após repouso de 15 minutos da paciente.

O risco cardiovascular foi calculado segundo o escore de Framinghan, somando-se os escores atribuídos às variáveis idade, níveis de colesterol total, HDL-C, glicemia de jejum, pressão arterial sistólica e diastólica e tabagismo. Este escore estabelece o risco de doenças cardiovasculares em 10 anos, sendo classificados em risco baixo 0 escore $<10$ (inferior a 10\%); risco moderado o escore entre II e 15 (de 17\% a 20\%) e risco alto o escore $>16$ (acima de 25\%) ${ }^{18}$.

Para análise dos dados, foram elaboradas estatísticas descritivas por meio de freqüências absolutas (n) e relativas (\%), cálculo das médias e desvio padrão e calculado o escore de Framingham, utilizando-se o programa SAS, versão 8.2 .

\section{Resultados}

A média de idade das mulheres foi de 56,5 \pm 5,7 anos; a média de idade na menopausa foi de $46 \pm 6,2$ anos; e o tempo médio de menopausa foi de $10,3 \pm 5,8$ anos. A maioria $(85,2 \%)$ das mulheres eram brancas, com escolaridade média de 4,9 $\pm 3,6$ anos, $67 \%$ não eram fumantes e $47 \%$ faziam terapia hormonal $(\mathrm{TH})$, sendo o tempo médio de uso da $\mathrm{TH}$ de 6,3 \pm 4,5 anos.

A somatória da frequêencia e duração das atividades físicas de cada seção do questionário IPAQ mostrou que 83,3\% das mulheres eram fisicamente ativas ou muito ativas (Tabela I).

Em relaçãa ao tipo de atividade física, as principais atividades relatadas por $92,6 \%$ das mulheres foram as tarefas domésticas dentro e fora de casa. Cerca de $71 \%$ das mulheres realizavam atividade física durante o transporte, sendo que 70,4\% eram caminhadas rápidas. Quanto à intensidade, houve predomínio de atividades de intensidade moderada (95,7\%) e caminhada (80,9\%). Apenas 32,1\% das mulheres referiram realizar caminhada como exercício físico e 6,8\% referiram praticar ginástica localizada durante o tempo de lazer (Tabela 2).

Os valores médios do colesterol total e frações, triglicérides, glicemia de jejum e pressões arteriais sistólica e diastólica apresentaram-se dentro de valores considerados normais (Tabela 3).

A maioria das mulheres apresentava escore de Framingham abaixo de 10 pontos, que é considerado risco baixo para doença cardiovascular, ou seja, inferior a 10\% de chances de ocorrer um evento cardiovascular em 10 anos. Em cerca de 5\% das mulheres, o 


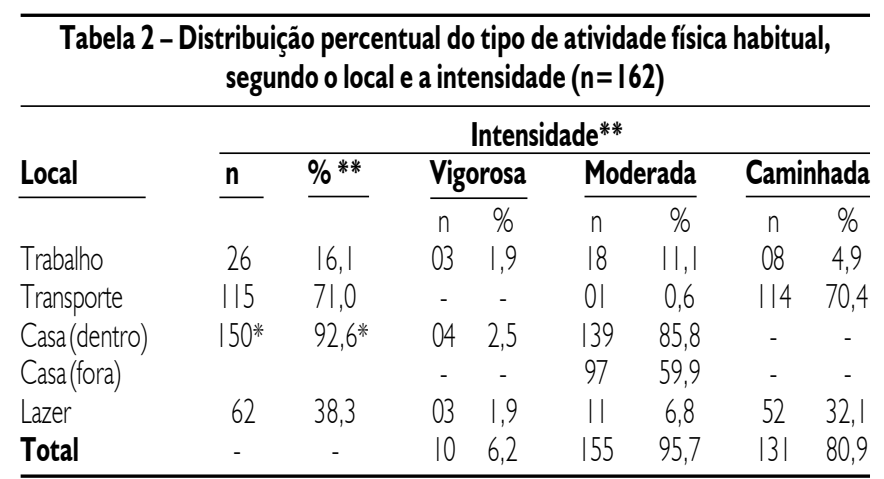

* Total de atividades físicas dentro e fora de casa

** Uma mesma mulher pode ter relatado mais de uma resposta em relação ao tipo e a intensidade da atividade física

Tabela 3 - Média dos parâmetros cardiovasculares em mulheres na pós-menopausa $(n=146)$

\begin{tabular}{|c|c|c|c|c|}
\hline Características & Média & DP & Mínimo & Máximo \\
\hline $\begin{array}{l}\text { Colesteroltotal (mg/dl) } \\
\operatorname{HDL}(\mathrm{mg} / \mathrm{dl}) \\
\mathrm{LDL}(\mathrm{mg} / \mathrm{dl}) \\
\text { Triglicérides (mg/dl) } \\
\text { Glicemiade jejum (mg/dl) } \\
\operatorname{PAS}(\mathrm{mmHg}) \\
\operatorname{PAD}(\mathrm{mmHg})\end{array}$ & $\begin{array}{l}211,0 \\
55,4 \\
138,3 \\
136,0 \\
96,3 \\
119,2 \\
77,3\end{array}$ & $\begin{array}{l}38,3 \\
14,8 \\
36,2 \\
71,6 \\
34,0 \\
15,4 \\
10,6\end{array}$ & $\begin{array}{l}124 \\
26 \\
47 \\
36 \\
28 \\
90 \\
50\end{array}$ & $\begin{array}{l}348 \\
119 \\
249 \\
494 \\
264 \\
200 \\
110\end{array}$ \\
\hline
\end{tabular}

Tabela 4 - Distribuição percentual do risco cardiovascular baseado no escore de Framinghan $(n=146)$

\begin{tabular}{lrr}
\hline Escore de Framingham & & $\frac{\mathbf{n}}{2}$ \\
\cline { 4 - 4 } Risco baixo (escore $\leq 10)$ & & $\frac{128}{87,7}$ \\
Risco moderado (escore II - I5) & 7 & 4,8 \\
Risco alto (escore $\geq 16$ ) & 11 & 7,5 \\
Total & 146 & 100 \\
\hline
\end{tabular}

escore apresentava entre $1 \mathrm{I}$ e 15 pontos, o que corresponde a um risco moderado (entre II\% e 20\% em 10 anos) e, em 7,5\%, o escore foi acima de 16 pontos, considerado risco alto, superior a 24\% em 10 anos (Tabela 4).

\section{Discussão}

Este estudo avaliou a prevalência e tipos de atividade física em mulheres na pós-menopausa. Os resultados mostraram que, nesta população, a maioria das mulheres era ativa, realizando predominantemente atividade física habitual como tarefas domésticas e caminhadas durante o transporte.

A prevalência da atividade física em mulheres na pós-menopausa foi de $83,3 \%$. Estes resultados foram bem maiores que os observados na população adulta dos EUA, que mostram um índice de 45,4\% de pessoas ativas², e na população na faixa etária de 30 a 69 anos no Estado de São Paulo observadas no estudo de Matsudo et al. ${ }^{4}$, que mostrou que menos de $50 \%$ das mulheres são ativas ou muito ativas. Entretanto, estudo realizado por Hallal et al. ${ }^{14}$, utilizando a versão curta do questionário IPAQ para avaliar o nível de atividade física em homens e mulheres de Pelotas-RS, mostrou uma prevalência de aproximadamente $60 \%$ de pessoas ativas. Dentre as mulheres na pós-menopausa, $62 \%$ com idade entre 40 e 59 anos e $57 \%$ entre 60 a 69 anos eram ativas.

Neste estudo, a maioria das mulheres (92,6\%) declarou realizar atividades em casa e no transporte (7|\%), sendo que poucas realizam alguma atividade física no trabalho $(16,1 \%)$. Coupland et al. ${ }^{19}$ também encontraram alta proporção de mulheres inglesas na pós-menopausa que realizam atividades em casa $(92,6 \%)$. Barros e Nahas ${ }^{20}$ verificaram em uma população de adultos jovens brasileiros que as atividades realizadas no trabalho e no lazer são as que exigem mais energia, sendo as atividades realizadas em casa e no transporte as que dispendem menor gasto energético.

A literatura tem mostrado uma grande variedade de instrumentos para quantificar a atividade física, o que tem dificultado a comparação dos resultados. O IPAQ foi elaborado com o propósito de criar um questionário-padrão que permitisse quantificar a atividade física em diferentes populações e que incluísse uma avaliação dos quatro componentes de atividade física. A maior prevalência de atividade física neste estudo, comparada à literatura, pode em parte ser atribuída ao uso de diferentes definições de atividade física, uma vez que o IPAQ avalia a quantidade total de atividades realizadas no trabalho, no transporte, em casa e no lazer, enquanto a maioria dos outros instrumentos restringe-se apenas às atividades realizadas no lazer. Hallal et al. ${ }^{14}$ enfatizam que, em países em desenvolvimento, como o Brasil, a ocupação e as atividades no transporte representam grande parte da atividade total dos indivíduos. Dessa forma, os dados reportados pelo IPAQ podem superestimar o nível de atividade física em comparação com outros instrumentos, podendo ser uma possível explicação para as diferenças encontradas. Além disso, o IPAQ é um dos poucos instrumentos que avalia a atividade física em casa, sendo a inclusão deste tipo de atividade um fator importante que pode ter contribuído para o aumento no nível de atividade encontrada, uma vez que as atividades domésticas são muito específicas e amplamente realizadas por mulheres nesta faixa etária.

$\mathrm{Na}$ população deste estudo, 38,3\% das mulheres faziam atividade física durante o lazer, sendo que 32, I\% caminhavam e 6,8\% faziam ginástica. A preferência por esses exercícios é semeIhante à encontrada no trabalho de Salles-Costa et al. ${ }^{21}$, que verificou maior aderência à caminhada e à ginástica em mulheres entre 20 e 60 anos de idade. Em relação à quantidade de exercício físico, também existe semelhança aos achados de Martinez-Gonzales et al. ${ }^{22}$, que verificaram uma baixa quantidade de atividade física durante o lazer, praticada nos países da União Européia. Dados brasileiros mostram que, nas regiões Sudeste e Nordeste do Brasil, 13\% das pessoas fazem atividade física durante o lazer', e entre as mulheres na cidade do Rio de Janeiro, este índice é de aproximadamente $22 \%{ }^{3}$.

Apesar de aproximadamente $81 \%$ das mulheres deste estudo referirem a caminhada como atividade física, apenas 32,1\% a praticavam dentro dos padrões recomendados. Estudos mostram que a caminhada realizada com duração, freqüência e intensidade adequadas torna-se um exercício benéfico para a saúde ${ }^{6}$ e pode ser 
uma opção de exercício físico que não demanda gasto financeiro. Simpson et al. ${ }^{23}$, acompanhando adultos americanos por 13 anos, verificou que a aceitabilidade da caminhada foi grande em todos os grupos socioeconômicos, e Pereira et al. ${ }^{24}$, acompanhando dois grupos de mulheres na pós-menopausa durante 10 anos, verificaram que o grupo que participava de caminhada permaneceu ativo em relação ao grupo que não caminhava, mostrando que pessoas que incluem atividades na sua rotina tendem a manter o padrão de atividades ao longo do tempo.

Estudos também têm mostrado que a atividade física está indiretamente relacionada ao nível socioeconômico, sendo que os indivíduos pertencentes à classe socioeconômica mais baixa tendem a realizar mais atividades ocupacionais e ter menos participação em atividades de lazer ${ }^{25}$. Os resultados deste estudo indicam diferenças entre atividade no lazer e atividade física total. Estudos que avaliam somente atividade no lazer tendem a subestimar o nível de atividade física em mulheres particularmente de menor nível socioeconômico' ${ }^{14}$, como na população estudada, uma vez que o baixo nível de escolaridade observado pode indiretamente estar relacionado a um baixo nível socioeconômico.

Em relação à intensidade das atividades, nesta população as mulheres realizavam pouca atividade de intensidade vigorosa, sendo a maioria atividade moderada. Discordando deste resultado, Hansen e Allen ${ }^{26}$ mostraram que. na população australiana, aproximadamente $50 \%$ das mulheres na pós-menopausa realizam atividade vigorosa. Evenson et al. ${ }^{27}$ avaliaram atividades vigorosa e moderada realizadas no tempo de lazer no presente e retrospectivamente nas idades de 18, 35 e 50 anos, e verificaram que, na pós-menopausa, há diminuição das atividades vigorosas, indicando que intervenções antes da menopausa podem ajudar a manter o padrão de atividade física durante a menopausa.

Uma possível limitação deste estudo pode ser relacionada à representatividade da amostra, por tratar-se de um grupo de mulheres na pós-menopausa freqüentadoras de um serviço de saúde, em que o contato periódico com profissionais de saúde, participação de grupos educativos e orientações quanto a hábitos de vida e à prática de atividade física são constantemente reforçados a cada consulta. Além disso, a utilização de questionários apresenta a limitação da subjetividade do instrumento e sua dependência da recordação precisa das atividades que podem ter sido supervalorizadas. Ainda assim, acreditamos que poderia ser maior o número de mulheres que aderissem a essas recomendações, visto que apenas pouco mais de um terço delas praticavam exercício físico.

Apesar da alta prevalência de atividade física habitual, houve baixa aderência ao exercício físico na população estudada. O risco de evento cardiovascular em dez anos, segundo escore de Framinghan, foi baixo para a maioria das mulheres. Esse baixo risco encontrado pode ser atribuído ao estilo de vida favorável e ao fato de tratar-se de um grupo de mulheres freqüentadoras de um serviço especializado, em que orientações quanto à dieta e importância da atividade física são constantemente reforçadas. Ainda assim, é importante ressaltar que aproximadamente $12 \%$ da população estudada apresentavam risco moderado ou alto de ocorrência de doença cardiovascular.

\section{Conclusão}

Entendemos que é necessário incentivar e orientar as mulheres na pós-menopausa a praticarem atividade física que seja compatível com suas condições físicas e financeiras, permitindo que esta população seja ativa não somente em relação à atividade física habitual, mas também em relação ao exercício físico. Apesar da idéia de que a atividade física habitual atua beneficamente na saúde, sabe-se que é preciso que esse tipo de atividade seja realmente praticada em quantidade, freqüência e intensidade ideais para melhora da aptidão física e promoção da saúde. Estudos avaliando a associação entre a prática de atividade física habitual e o impacto sobre a aptidão física são necessários para saber se este tipo de atividade é suficiente para produzir os mesmos benefícios em relação à saúde alcançados pelo exercício físico.

\section{Agradecimento}

Fundação de Amparo à Pesquisa do Estado de S. Paulo - FAPESP/ Processo n $03 / 01423-0$.

\section{Conflito de interesse: Não há}

\section{SUMMARY}

Habitual PHYSICAL activity aNd CARDIOVASCULAR RISK IN POST MENOPAUSE

BACKGROUND. To evaluate the prevalence of habitual physical activity and cardiovascular risk in postmenopausal women.

METHODS. A cross-sectional study was made of 162 women, aged 40 65 years, amenorrheic for at least 12 months, receiving care at the menopause clinic ofCAISM/UNICAMP. The women verbally answered the International Physical Activity Questionnaire for assessment of physical activity levels. This instrument evaluates the frequency and duration of activities performed during transportation, at work, at home or in leisureover the course of a normal week. Women were classified as sedentary, insufficiently active, active orvery active. Laboratory tests were carried out to measure total serum cholesterol, high density lipoproteins and fasting glucose. Systolic and diastolic pressure was measured.

RESULTS. The mean age of women in this study was 56.5 years and mean age at menopause was 46 years. Prevalence of physical activity was $83.3 \%$ of which $80.8 \%$ were classified as active, $2.5 \%$ as very active and $16.7 \%$ as insufficiently active. Principal activities were performed during transportation and while carrying out household chores. Only $38.3 \%$ of women had leisure-time physical activities. In the population, $87.7 \%$ of the women presented a Framingham score $<10$, considered of low cardiovascular risk.

Conclusions. Postmenopausalwomen showa high prevalence of habitual physical activity and low adherence to physical exercise. Cardiovascular risk was low in the population studied. It is important to encourage these women to practice physical activity compatible with their physical condition, to orient and stimulate them to practice not only their habitual physical activity but also physical exercise. [Rev Assoc Med Bras 2006; 52(4): 242-6]

KEY WORDS: Motor activity. Exercise. Cardiovascular risk. Post menopause. 


\section{REFERÊNCIAS}

I. Monteiro CA, Conde WL, Matsudo SM, Matsudo, VR, Bonseñor IM, Lotufo, PA. A descriptive epidemiology of leisure-time physical activity in Brazil, 1996-1997. Rev Panam Salud Publica/Pan Am J Public Health 2003; | 4:246-54.

2. Macera CA, Jones DA, Yore MM, Ham AS, Kohl, HW, Kimsey CD, et al. Prevalence of physical activity, including lifestyle activities among adults: United States, 2000-2201.MMWR Morb Mortal Wkly Rep 2003; 52:764-69.

3. Gomes VB, Siqueira KS, Sichier, R. Atividade física em uma amostra probabilística da população do Município do Rio de Janeiro. Cad Saúde Pública 200 I; 17:969-76.

4. Matsudo SSM, Matsudo VKR, Araújo T. Nível de atividade física da população do Estado de São Paulo: Análise de acordo com o gênero, idade, nível sócio-econômico, distribuição geográfica e de conhecimento. Rev Bras Cienc Mov 2002; 1 0:41-50.

5. Ciolac EG, Guimarães GV. Exercício físico e síndrome metabólica. Rev Bras Med Esporte 2004; 10:319-24.

6. Asikainen TM, Miilunpalo S, Oja P, Rine M, Passanen M, Vuori I. Walking trials in postmenopausal women: effect of one vs two daily bouts on aerobic fitness. Scand J Med Sci. Sports 2002; I 2:99- 105.

7. Monteiro MF, Sobral Filho, DC. Exercício e o controle da pressão arterial. Rev Bras Med Esporte 2004; 10:6:5 I3-6.

8. Thompson PD, Buchner D, Piña IL, Balady GJ, Williams MA, Marcus $\mathrm{BH}$, et al. Exercise and physical activity in the prevention and treatment of atherosclerotic cardiovascular disease: a statement from the Council on Clinical Cardiology (Subcommittee on Exercise, Rehabilitation and Prevention) and the Council on Nutrition, Physical Activity, and Metabolism (Subcommittee on Physical Activity). Circulation 2003; 107:3109-16.

9. Pate RR, Pratt M, Blair SM, Haskell WL, Macera CA, Bouchard C, et al. Physical activity and public health: a recommendation from the Centers for Disease Control and Prevention and the Americam College of Sports Medicine. JAMA 1995;273:402-7.

I0. Carvalho T, Nóbrega ACL, LazzoliJK, Magni JRT, Rezende L, Drummond FA, et al. Posição oficial da Sociedade Brasileira Medicina do Esporte: atividade física e saúde. Rev Bras Med Esporte 2001;7:79-81.

I I. Leitão MB, LazzoliJK, Oliveira MAB, Nóbrega ACL, Silveira GG, Carvalho $\mathrm{T}$, et al. Posicionamento Oficial da Sociedade Brasileira de Medicina do Esporte: atividade física e saúde da mulher. Rev Bras Med Esporte 2000;6:215-20.

12. Piché ME, Weisnagel SJ, Corneau L, Nadeau A, Bergeron J, Lemieux S. Contribution of abdominal visceral obesity and insulin resistance to the cardiovascular risk profile of postmenopausal women. Diabetes 2005;54:770-7

13. Hunter GR, Weinseir RL, Zuckerman PA, Darnell BE. Aerobic fitness, physiologic difficulty and activity in black and white women. Int J Obes Relat Metab Disord 2004;28: I I I I-7.
14. Hallal PC, Victora CG, Wells JCK, Lima RC. Physical Inactivy: Prevalence and associated variables in brazilian adults. Med Sci Sports Exerc 2003;35: I 894-900.

15. Thompson SK. Sample size for estimating multinomila proportions. Am Stat 1987;41:42-6.

16. Craig CL, Marshall AL, Sjöström M, Bauman AE, Booth ML, Ainsworth BE, et al. International physical activity questionnaire: 12: country reliability and validity. Med Sci Sports Exerc 2003;35: I 38 I-95.

17. Matsudo SSM, Araújo T, Matsudo VKR, Andrade D, Andrade E, Oliveira LC, et al. Questionário Internacional de Atividade Física (IPAQ), estudo de validade e reprodutibilidade no Brasil. Rev Ativ Fis Saúde 200 I;6:5-18.

18. Anderson KM, Odell PM, Wilson PWF, Kannel WB. Cardiovascular disease risk profiles. Am. Heart J 1990; 121 :293-8.

19. Coupland CAC, Cliffe SJ, Bassey EJ, Grainge MJ, Hosking DJ, Chilvers CED. Habitual physical activity and bone mineral density in postmenopausal women in England. Int J Epidemiol 1999;28:24I-6.

20. Barros MVG, Nahas MV. Reprodutibilidade (teste-reteste) do questionário international de atividade física (QIAF-Versão 6): um estudo piloto com adultos no Brasil. Rev Bras Cienc Mov 2000; 1:23-6.

21. Salles-Costa R, Heilborn ML, Werneck GL, Faerstein E, Lopes CS. Gênero e prática de atividade física de lazer. Cad Saúde Pública 2003; 19:325-33.

22. Martinez-Gonzalez MA,Varo JJ, Santos JL, Irala J, Gibney M, Kearney J, et al. Prevalence of Physical activity during leisure time in the European Union. Med Sci Sports Exerc 200 I;33: I | $42-6$.

23. Simpsom ME, Serdula M, Galuska DA, Gillespie C, Donehoo R, Macera C, et al. Walking trends among U.S. adults: the behavioral risk factor surveillance system, 1987-2000. Am J Prev Med 2003;25:95- 100.

24. Pereira MA, Kriska AM, Day RD, Cauley JA, LaPorte RE, Kuller LH. A randomized walking trial in postmenopause women. Arch Intern Med 1998; 158:1695-701.

25. Cauley JA, Donfield SM, LaPorte RE, Warhaftig NE. Physical Activity by socioeconomic status in two population based cohorts. Med Sci Sports Exerc 1991;23:343-51.

26. Hansen R, Allen BJ. Habitual physical activity, anabolic hormones and potassium content of fat-free mass in postmenopausal women. Am J Clin Nutr 2002;75:3| 4-20.

27. Evenson KR, Wilcox S, Pettinger M, Brunner R, King AC, McTiernan A. Vigorous leisure activity through women's adult life. Am J Epidemiol 2002; 156:945-53. 